\title{
Reflexiones sobre el estado de flujo de efectivo e ideas para su presentación por el método directo
}

\author{
Reflections upon the statement of cash flow and ideas \\ for its presentation using the direct method \\ Reflexões sobre a demonstração dos fluxos de caixa e ideias \\ para apresentação pelo método direto \\ Gustavo Roberto Rondi \\ Facultad de Ciencias Económicas \\ y Sociales de la Universidad Nacional \\ de Mar del Plata, Argentina. \\ E-mail: gustavorondi@gmail.com \\ María del Carmen Casal \\ Facultad de Ciencias Económicas \\ y Sociales de la Universidad Nacional \\ de Mar del Plata, Argentina. \\ E-mail:carmencasal2010@gmail.com \\ Marcelo Javier Galante \\ Facultad de Ciencias Económicas \\ y Sociales de la Universidad Nacional \\ de Mar del Plata, Argentina. \\ E-mail:marcelogalante@galanteyaso- \\ ciados.com.ar \\ Melisa Gómez \\ Facultad de Ciencias Económicas \\ y Sociales de la Universidad Nacional \\ de Mar del Plata, Argentina. \\ E-mail:melisagomezcp@gmail.com
}

Fecha de recepción: 22/03/2017 Fecha de aceptación: 22/05/2017

\section{Resumen}

A los fines de efectuar proyecciones y análisis financieros, la información contenida en el estado de flujo de efectivo debe complementarse con el análisis de los saldos de activos y pasivos operativos vinculados, por lo cual cobra relevancia el método seleccionado para confeccionar el estado.

Si se utiliza el método directo para la presentación del flujo de efectivo de las actividades operativas, la lectura y comprensión del estado por parte del usuario es sencilla, pero no es suficiente para el análisis de la vinculación entre el resultado y la generación de fondos del periodo, aspecto fundamental para realizar proyecciones financieras. La presentación por el método indirecto, permite exponer dicha vinculación, pero resulta de difícil comprensión.

Palabras clave

- Flujo de efectivo

- Resultado operativo

- Desempeño

- Rentabilidad

En este trabajo proponemos dos alternativas para presentar el estado por el método directo, que consolidan las ventajas del mismo en su versión tradicional, con las del método indirecto. Las propuestas incluyen la exposición de información en el cuerpo del estado o en nota complementaria, 
detallando cómo se determinan las distintas clases de entradas y salidas brutas de efectivo que se exponen por el método directo, partiendo de los resultados devengados y considerando los aumentos 0 disminuciones de activos y pasivos operativos vinculados.

El método directo favorece el análisis vertical del estado de flujo de efectivo y el horizontal a lo largo de dos o más periodos. Asimismo, resulta útil la información que puede obtenerse mediante el cálculo de ratios que vinculan los distintos subtotales de este estado y con algunos del estado de resultados.

\begin{abstract}
In order to carry out financial projections and analysis, the information contained in the statement of cash flow should be supplemented by the analysis of the balances of related operating assets and liabilities, which is why the method selected to prepare the statement becomes relevant.

If the direct method is used for the presentation of the cash flow, the reading and understanding of the statement by the user is simple, but it is not sufficient for the analysis of the link between the result and the generation of funds of the period - a fundamental aspect for making financial projections. The presentation using the indirect method can also show such connection, but it is difficult to understand it.

In this paper, we propose two alternatives to present the statement by the direct method, which consolidate the advantages of its traditional version with those of the indirect method. The proposals include the disclosure of information in the body of the accounting statement or in a supplementary note, describing in detail how the different types of inflows and outflows of cash that are exposed by the direct method are determined, based on the accrued results, and considering the increases or decreases of related operating assets and liabilities.

The direct method favors the vertical and horizontal analysis of the cash flow statement over two or more periods. The information that can be obtained by calculating ratios that link the different subtotals of this statement and some of the income statement also proves to be useful.
\end{abstract}

Keywords

- Cash Flow

- Operating Result

- Performance

- Profitability

\title{
Resumo
}

A fim de fazer projeções e análises financeiras, a informação contida na demonstração de fluxo de caixa deve ser complementada com a análise dos saldos de ativos e passivos operacionais ligados, pelo que se torna relevante 0 método selecionado para fazer a demonstração.

Se é utilizado o método direto para a apresentação do fluxo de caixa das atividades operacionais, a leitura e compreensão da demonstração para 0 usuário é simples, mas não é suficiente para a análise da relação entre 0 
Palavras-chave

- Fluxo de caixa

- Resultado operacional

- Desempenho

- Rentabilidade resultado e a geração de fundos do período, aspecto essencial para fazer projeções financeiras. A apresentação pelo método indireto, permite expor essa ligação, mas apresenta dificuldades na compreensão.

Neste trabalho, propomos duas alternativas para a apresentação da demonstração pelo método direto, que consolidam as vantagens do mesmo em sua versão tradicional, com as do método indireto. As propostas incluem a exposição de informação no corpo da demonstração ou em nota complementar, detalhando como se determinam os diferentes tipos de entradas e saídas brutas de caixa que são expostos pelo método direto, partindo dos resultados acumulados e considerando os aumentos ou diminuições de ativos e passivos operacionais vinculados.

0 método direto favorece a análise vertical da demonstração dos fluxos de caixa e a horizontal ao longo de dois ou mais períodos. Além disso, é útil a informação que se pode obter por meio do cálculo de rácios que vinculam os diferentes subtotais desta demonstração e com alguns da demonstração do resultado do exercício.

\section{Introducción}

Los estados contables deben proveer información sobre el patrimonio del ente emisor y su evolución en el período que abarcan, a fin de que los usuarios de los mismos puedan emplearlos como un elemento (aunque no el único) para la toma de sus decisiones. Dichos estados incluyen información sobre composición del patrimonio, las causas del resultado devengado en el período, y evolución y origen de los recursos financieros, información que será empleada, entre otros usos, para evaluar la gestión de los administradores, predecir los resultados futuros, comparar el desempeño del emisor con el de otros, tomar decisiones societarias sobre distribución de dividendos y fijar remuneraciones de los órganos de administración, y como base para determinaciones impositivas.

Al definir el contenido de los estados contables se tienen en cuenta las necesidades de los llamados usuarios tipo (Fowler Newton, 2011:26-27), que les permitan obtener información acerca del desempeño del ente y analizar en particular, entre otras cuestiones:

1. La capacidad para cancelar sus obligaciones y distribuir ganancias;

2. El origen, naturaleza y rendimiento de los recursos que emplea;

3. La rentabilidad, solvencia y capacidad de crecimiento y a partir de ello la gestión de los administradores.

La información acerca del patrimonio se brinda mediante el Estado de Situación Patrimonial. Los restantes estados contables básicos informan acerca de la evolución del patrimonio, desde el punto de vista económico y financiero. La evolución económica del patrimonio se informa a través del estado de resultados y del estado de evolución del patrimonio neto. En el primero se exponen las 
causas del resultado atribuible al período, mediante la presentación de los ingresos, gastos, ganancias y pérdidas devengados; mientras que en el segundo se exponen las otras causas de cambios en la riqueza del ente, principalmente generadas por aportes y retiros de los propietarios, aunque también puede incluir ciertos resultados que no han sido informados como tales en el estado de resultados y que se exponen directamente en este estado porque así lo exigen disposiciones normativas. La evolución financiera del patrimonio, en particular de los recursos financieros, se informa a través del estado de flujo de efectivo, que expone la cuantía en que se vio modificado el efectivo y equivalentes de efectivo del ente a lo largo del periodo que abarcan los estados contables y las causas que generaron dicha modificación.

Uno de los principales usuarios de los estados contables son las entidades financieras, que analizan fundamentalmente la capacidad de pago de las obligaciones por parte del ente emisor de los estados, a fin de definir el otorgamiento de asistencia financiera. En este caso cobra fundamental importancia la fortaleza del flujo de fondos, junto con la estructura patrimonial y la rentabilidad puestas en evidencia en los estados de situación patrimonial y de resultados, respectivamente. De todas maneras, la generación de efectivo o recursos financieros está directamente vinculada (o debería estarlo) con la rentabilidad. En consecuencia, a los fines de analizar el desempeño del ente y en particular su capacidad para cumplir con sus obligaciones y distribuir utilidades, el estado de resultados y el estado de flujo de efectivo se complementan, y deberían ser analizados en conjunto.

Sin embargo, conforme la información requerida por la normativa vigente en nuestro país en relación con ambos estados, consideramos que el análisis conjunto se dificulta. Consecuentemente, mediante el presente, proponemos algunas soluciones para que esta vinculación entre ambos estados sea más clara y por ende más útil, atento a las necesidades de los usuarios de la información contable.

\section{Análisis del desempeño del ente: ¿estado de resultados o estado de flujo de efectivo?}

\subsection{Estado de resultados}

El estado de resultados informa acerca del resultado atribuible a un periodo y las causas que lo generaron. Definimos el resultado como la variación operada en el patrimonio de un ente a lo largo de un periodo y que no ha sido generada por transacciones con los propietarios. Este concepto se condice con el criterio de capital a mantener financiero para determinar el resultado de un periodo y con la información que se brinda en el estado de resultado integral, cuya presentación es requerida por las Normas Internacionales de Información Financiera (NIC 1) (IASB, 2016) y por la NIIF para las Pymes (IASB, 2015). No obstante, este estado no es exigido por la normativa nacional que solo requiere la presentación del estado de resultados, el cual no incluye los denominados otros resultados integrales, que son aquellos que, aunque devengados en el periodo por el cual se informa, no se exponen en el estado de resultados, sino en el estado de evolución de patrimonio neto. Consideramos necesario que las normas nacionales requieran que se informe acerca del resultado integral tal como se concibe en las normas internacionales, posición que manifestamos en otra presentación, a la que remitimos para profundizar los fundamentos (Rondi, Casal, Galante y Gómez, 2014).

Si bien no es pretensión de este trabajo enfatizar en el análisis del estado de resultados, nos parece oportuno mencionar que a tal efecto se utilizan habitualmente diversos indicadores, tales como:

- Rentabilidad del activo: Resultado generado por el activo/Promedio del activo.

- Rentabilidad del capital empleado (ROCE): Resultado operativo neto de impuesto a las ganancias / Activo afectado a actividades principales menos Pasivo no remunerado.

- Rentabilidad de la inversión: Resultado del período/ Promedio del patrimonio neto. 
El subtotal Resultado Operativo surge de considerar el resultado bruto, que surge de deducir a los ingresos por ventas de bienes o servicios el costo de tales bienes vendidos o servicios prestados; menos gastos operativos de administración, comercialización y otros; y, si los hubiere, resultados por participaciones en entes con actividad vinculada a la del emisor de los estados. La presentación de este subtotal no es requerida por las normas contables profesionales vigentes. No obstante, es habitualmente presentada por entes que cotizan en bolsa.

También es recurrente la presentación de información acerca de la EBITDA en memorias de administradores, la que se considera como un indicador de desempeño y como una aproximación al flujo de efectivo operativo de la empresa. EBITDA es la sigla de la expresión en inglés Earning Before Interest, Taxes, Depreciation and Amortization: ganancia antes de intereses, impuesto a las ganancias, depreciaciones y amortizaciones. Este indicador ha sido objeto de variadas críticas. Además de la falta de criterio uniforme en su determinación, se sostiene que si lo que se quiere reflejar es la generación de efectivo de las actividades operativas, debería recurrirse al estado de flujo de efectivo y no emplear este indicador simplificado. Asimismo, se ha argumentado que como ratio económico no es sólido, por no incluir en su determinación a las amortizaciones y depreciaciones, ya que no parece razonable suponer que no sería necesario adquirir bienes de uso 0 activos intangibles para poder llevar adelante la actividad generadora de ingresos. № obstante, creemos que puede ser un buen indicador de la incidencia en el flujo de efectivo de las actividades operativas, una vez que se hayan cobrado y pagado todos los ingresos y gastos operativos devengados, antes del impuesto a las ganancias y los cargos por intereses. Para ampliar este tema puede consultarse Rondi y Galante (2007).

Si se pretendiera arribar al flujo de efectivo de las actividades operativas partiendo de la EBITDA, habría que considerar también otros resultados que no tienen efecto sobre el efectivo, agregar los cambios en el capital operativo producidos en el período (aumentos 0 disminuciones en créditos por ventas, existencias de bienes de cambio, proveedores, etc.), deducir los pagos del impuesto a las ganancias y, según la opción de exposición tomada por el ente, tomar en cuenta los pagos de intereses y dividendos. Los autores consultados proponen el siguiente cálculo a los fines de efectuar una conciliación entre ambos conceptos:

\begin{tabular}{l}
\hline Resultado del activo \\
\hline Más: Depreciaciones y amortizaciones \\
\hline EBITDA \\
\hline Más (menos) otras partidas de resultado \\
sin efecto sobre el efectivo (a) \\
\hline Menos (más) aumento (disminución) capital operativo \\
\hline Menos: pagos de intereses (b) \\
\hline Menos: pagos del impuesto a las ganancias \\
Flujo neto de efectivo proveniente \\
de las actividades operativas
\end{tabular}

a) Algunos entes consideran estas partidas para determinar la EBITDA.

b) Esta línea no se expondría en el esquema si el ente hubiera optado por considerarla dentro de las actividades de financiación.

Por otra parte, suelen calcularse e informarse algunos ratios o indicadores a partir de la EBITDA, por ejemplo:

- EBITDA / Ventas. Se utiliza para comparar el desempeño de distintos entes del mismo sector y para ver su evolución en el propio emisor de los estados. Indica que porción de los ingresos operativos queda disponible para afrontar el pago de intereses, impuesto a las ganancias, reposiciones de activo fijo y distribuciones de ganancias.

- EBITDA / Intereses. Cuanto más alto es este valor, mejor es la capacidad financiera de la empresa para afrontar el pago de los intereses. Cuando su valor se acerca a 1 (uno), es indicativo de que el ente puede estar teniendo dificultades financieras. 
- Deuda Financiera / EBITDA. Utilizado para analizar la salud financiera de un ente. Cuando más reducido sea su valor, mejor es la situación financiera de la empresa. Indica en cuantos años se cancelaría la deuda financiera con la EBITDA.

- Valor de la Empresa / EBITDA El valor de la empresa se determina sumando patrimonio neto y deuda financiera. Informa en cuantos años se recuperaría el valor de la empresa con su EBITDA.

\subsection{Estado de flujo de efectivo}

El estado de flujo de efectivo brinda información acerca de:

- la variación producida en el efectivo y equivalentes de efectivo de un ente a lo largo de un periodo determinado, definiéndose a estos últimos, como aquellos que se mantienen con el fin de cumplir con los compromisos de corto plazo más que con fines de inversión, y

- las causas que generaron dicha variación, las cuales se clasifican en producidas por actividades operativas, de inversión y de financiación. Eventualmente algunos conceptos podrán informarse en una cuarta causa denominada Resultados financieros y por tenencia generados por el efectivo y equivalentes de efectivo (FACPCE, 2008).

Los equivalentes de efectivo son inversiones de alta liquidez fácilmente convertibles en efectivo y que están sujetas a riesgos insignificantes de cambios de valor.

Los cuerpos normativos aplicables a los distintos entes domiciliados en nuestro país, contienen requerimientos similares y no presentan diferencias sustanciales en relación con la información a ser suministrada por este estado (Rondi, Casal, Galante y Gómez, 2014). Consecuentemente, a lo largo del presente trabajo, no haremos referencia a ninguna norma en particular, ya que en relación con los aspectos que abordaremos, todas ellas sostienen iguales criterios.
Este estado adopta un concepto líquido de fondos, posibilitando en principio analizar y proyectar la capacidad del ente para hacer frente a la cancelación de sus obligaciones y compromisos y para la distribución de utilidades, las cuales se realizan en su mayor parte en efectivo.

No obstante, para poder realizar una proyección en relación con la capacidad del ente para generar recursos financieros, debemos necesariamente complementar el análisis de este estado con la información contenida en el resto de los estados contables, ya que el estado de flujo de efectivo por sí mismo, resulta ser insuficiente. Compartimos lo manifestado por Fowler Newton (2001:690) en cuanto que «la capacidad de un ente para afrontar sus compromisos y distribuir ganancias depende en última instancia de su rentabilidad. Por lo indicado (...) deben considerarse también los resultados de Ios análisis de la estructura patrimonial, del capital corriente y de la rentabilidad».

El estado de flujo de efectivo pone de manifiesto estrategias y políticas del ente en materia de inversiones, fuentes de financiamiento, decisiones vinculadas con ampliación o reducción de plazos de crédito comercial, stocks, entre otras cuestiones. La adecuada interpretación del mismo en conjunto con los restantes estados financieros básicos, puede permitir proyectar la evolución financiera futura, comprobar la alineación de la administración 0 gestión de fondos con los objetivos globales del ente, determinar las causas de problemas financieros e inclusive anticiparlos, pudiendo ser empleado como una herramienta de gestión. Por ende, entendemos que la información que brinda es de suma utilidad para el usuario, más allá de ser requerida su presentación con carácter obligatorio por parte de todos los cuerpos normativos vigentes en nuestro país.

Por otra parte, su análisis permite alertar acerca de variaciones significativas de determinados activos o pasivos operativos, en relación con la generación de fondos, que puedan poner en peligro a la entidad en el corto o mediano plazo. 


\subsubsection{Análisis del estado de flujo de efectivo y su vinculación con el resultado del periodo}

La información acerca de la variación operada en el monto de los fondos desde el inicio de un periodo hasta su finalización, no es necesariamente significativa para definir una situación financiera favorable o desfavorable o para evaluar la gestión de fondos del ente, ya que podrían producirse variaciones bruscas en muy cortos periodos de tiempo, que inclusive lleven al usuario a conclusiones erróneas (Fowler Newton, 2001:688). Así, por ejemplo, si se han producido retrasos considerables en las cobranzas del principal cliente, por cuestiones circunstanciales, se podrán mostrar escasos recursos generados en un periodo, y abultados ingresos generados en el siguiente, en el cual opera la cobranza completa.

Por otro lado, también podría prima facie ponerse de manifiesto una situación financiera favorable generada, por ejemplo, por el incumplimiento de obligaciones, 0 una situación financiera desfavorable originada por el alargamiento de los plazos de cobranzas. Por lo tanto, en ambos casos, a los fines de efectuar proyecciones y análisis financieros, la información contenida en el estado de flujo de efectivo no es suficiente, aun mediante el análisis de sus causas, y debe necesariamente, complementarse con el análisis de los saldos de los activos y pasivos operativos vinculados. Es así, que podría generarse un flujo de fondos positivo, que en sí mismo no puede ser considerado como una evidencia de situación financiera favorable, dicho flujo positivo debería ser analizado en relación con su origen, tomando en cuenta situaciones tales como:

- si el mismo se origina principalmente en cobranzas de periodos anteriores y se ha producido una disminución de las ventas del periodo,

- si el mismo se ha generado mediante cobranzas de ventas del periodo, las cuales inclusive se han incrementado en relación con periodos anteriores.

Es factible que en ambos casos la generación de efectivo por cobros por ventas sea la misma, pero la proyección a futuro es sustancialmente diferente en uno y otro caso, atento a las fuentes que han generado el incremento del efectivo.

En este punto cobra relevancia el método seleccionado para brindar información en relación con los flujos de efectivo y equivalentes de efectivo generados por las actividades operativas. Recordemos que los mismos pueden exponerse mediante el método directo 0 el indirecto. En este grupo de actividades se reflejan los efectos financieros de los resultados operativos del ente, de allí que, a los fines de su vinculación con el estado de resultados proponemos se informe en el mismo el subtotal de resultados operativos, tal como lo exponen la mayor parte de las entidades que cotizan sus acciones en bolsa.

Mediante el método directo se informan las principales clases de entradas y salidas brutas de efectivo y equivalentes, de manera tal que su lectura y comprensión por parte del usuario es sencilla, en particular a los fines de realizar proyecciones. Sin embargo, no resulta ser suficiente para el análisis de la vinculación del resultado obtenido por el ente y la generación de fondos del periodo, e inclusive puede inducir a error en su interpretación, de acuerdo con lo que planteamos en párrafos anteriores. Un flujo positivo de fondos puede haberse generado, por ejemplo, por cobranzas de saldos de periodos anteriores o por incumplimientos de obligaciones, sin que ello se vea reflejado en el estado presentado mediante este método, lo que hace necesario recurrir a su análisis conjunto con los saldos patrimoniales vinculados. En consecuencia, podemos afirmar que la información brindada por este método no muestra los efectos en el flujo de fondos generado por variaciones patrimoniales significativas y que impactan favorable 0 desfavorablemente en el corto y mediano plazo.

En el método indirecto, en cambio, se parte del resultado del periodo segregado en ordinario y extraordinario, conforme se informa en el estado de resultados, y se suman o restan partidas de ajuste o conciliación, para arribar al flujo de efectivo generado por actividades operativas. Las partidas de conciliación son: 
- las que integran el resultado del periodo, pero no afectaron ni afectarán el flujo de efectivo 0 equivalentes de efectivo;

- aquellas que conforman el resultado del periodo, pero cuyos flujos de efectivo 0 equivalentes deben ser informados en actividades de inversión o financiación;

- las que forman parte del resultado del periodo corriente, pero tendrán incidencia en el efectivo 0 equivalentes en periodos siguientes;

- aquellas que integraron el resultado de periodos anteriores, pero tienen incidencia en el efectivo 0 equivalentes del periodo corriente.

Estos dos últimos grupos de partidas de conciliación son los vinculados con las variaciones patrimoniales a las que hacíamos referencia. Si bien la lectura y comprensión de la información presentada por este método es más compleja para el usuario, permite explicar la vinculación entre el resultado del periodo y los flujos de efectivo y equivalentes generados por las actividades operativas.

Este es justamente el método que vincula ambos estados, el de resultados y el estado de flujo de efectivo, en un proceso de análisis del desempeño del ente. Cabe señalar que la mayoría de las entidades que cotizan sus títulos en bolsa, presentan sus estados aplicando el método indirecto. Esta evidencia es contraria a las posturas doctrinarias que en forma mayoritaria se pronuncian a favor de la utilización del método directo para exponer los flujos de fondos de las actividades operativas. Sería interesante relevar información de los emisores acerca de los motivos por los cuales prefieren utilizar el método indirecto.

A los fines de que la información expuesta favorezca esta vinculación y que al mismo tiempo sea de fácil comprensión para el usuario de los estados contables, proponemos efectuar una apertura de las partidas simples que se presentan en el método directo, explicitando el cálculo mediante el cual los resultados devengados e informados en el estado de resultados, se «convierten» en partidas cobradas o pagadas según corresponda. Así, por ejemplo, a los fines de informar el monto de efectivo generado por los cobros por ventas, deberíamos partir de las ventas del estado de resultados, sumarle las cobranzas por los saldos iniciales de créditos por ventas y restarle los importes de ventas no cobrados puestos de manifiesto en los saldos finales de créditos por ventas. Esta apertura podrá efectuarse mediante notas a ser presentadas como información complementaria, o podrá formar parte del cuerpo del estado. Más adelante presentamos un ejemplo en el cual abordamos las dos alternativas. De esta manera, se pone de manifiesto la relación entre los estados de resultados y de flujo de efectivo, considerando que la apertura de la información propuesta es el puente que los vincula, quizás con mayor claridad que la requerida por el método indirecto. Asimismo, permite mejorar sustancialmente el análisis y la proyección a efectuar, teniendo en cuenta la incidencia de los saldos de los rubros patrimoniales correspondientes.

\subsubsection{Análisis del estado de flujo de efectivo en relación con las distintas causas generadoras de fondos. Análisis vertical}

En la medida en que la información se presente utilizando el método directo, es posible efectuar un análisis vertical del estado de flujo de efectivo determinando la incidencia o participación de las distintas partidas del estado en relación con los subtotales o totales del mismo. De la misma manera, podríamos realizar un estudio horizontal, 0 de evolución de las partidas a lo largo de dos 0 más periodos.

Algunos autores proponen además, el cálculo de ratios vinculando entre sí los distintos subtotales de este estado y con algunos del estado de resultados (Pérez, 2008). Es necesario aclarar que es conveniente realizar este análisis a través de diversos periodos, a fin de verificar la consistencia entre la gestión financiera y la estrategia global del ente, y mediante un previo estudio en detalle de sus componentes para evitar arribar 
a conclusiones erróneas. Ya hemos manifestado que un flujo de efectivo positivo o negativo no es en sí mismo evidencia de una situación financiera favorable 0 desfavorable ni de que se haya desarrollado una buena gestión de fondos. Por lo tanto, debería profundizarse el análisis de los orígenes 0 fuentes que generaron efectivo $\mathrm{y} / 0$ de los destinos a los cuales el efectivo se haya aplicado. Incluso es necesario poder determinar la etapa del ciclo de vida que está atravesando la empresa, teniendo en cuenta que los requerimientos 0 excedentes de fondos difieren, sin que sean en sí mismos buenos 0 malos indicios en relación con la gestión financiera. Es así, que en una etapa inicial o de crecimiento emergente es factible que exista una necesidad de fondos a ser aplicados a actividades operativas, y que para ello se recurra a fuentes de financiamiento externas. El ente tiene una necesidad de recursos que no puede ser totalmente cubierta por los fondos generados por sus actividades, y se convierte en un «consumidor de efectivo» (García Fronti, 2003). En otras etapas esta misma situación podría poner en evidencia falencias en la gestión de fondos 0 problemas financieros.

A los fines de adentrarnos en el análisis del estado, debemos considerar las relaciones entre los flujos de fondos procedentes de las distintas actividades entre sí 0 de las diferentes partidas dentro de cada una de ellas.

Así, por ejemplo, si analizamos las variaciones de efectivo producidas por las actividades operativas, deberíamos verificar si los cambios favorables provienen de resultados operativos a ser sostenidos en el tiempo, qué tipo de variaciones se han producido en los activos y pasivos operativos, si se han presentado ampliaciones en los plazos de cobranzas 0 de pago, etc. Un incremento en los pagos a proveedores, proveniente de un incremento en los stocks, 0 un aumento en los plazos de cobranza (consecuencia por ejemplo de una política de expansión 0 de ingreso a nuevos mercados) generan una reducción del efectivo, que no necesariamente pone en evidencia una situación financiera desfavorable, y debe ser analizada en el contexto de los restantes estados y de las políticas de la empresa.

De la misma forma, debería procederse para el análisis de los flujos de efectivo provenientes de las actividades de financiación, en donde cobra fundamental importancia verificar el destino de los flujos positivos de fondos. Si, por ejemplo, fueran requeridos para hacer frente a gastos operativos, se pone en evidencia un estado financiero delicado que, de mantenerse en el tiempo, pondría al ente en situación de riesgo. En cambio, sustancialmente diferente sería si se destinaran a la inversión en activos productivos, situación típica en etapas de expansión 0 crecimiento. Cualquiera sea el caso, adicionalmente, se deberán tener en cuenta, los futuros compromisos de fondos que se derivarán de estas financiaciones.

De igual manera, se deberán analizar las causas por las cuales se produce un flujo negativo en las actividades de financiación y, en particular, de qué manera se han obtenido los fondos para hacer frente a esta disminución. Si provienen de actividades operativas, se pondría de manifiesto la capacidad del ente para generar los recursos suficientes para hacer frente a sus obligaciones 0 al pago de dividendos; en cambio, si el ente se vio obligado a desprenderse de activos fijos, podría ser reflejo de una situación comprometida, no sólo por la incapacidad de generar fondos sino también por la reducción de la estructura productiva.

Al analizar los flujos de efectivo provenientes de las actividades de inversión, deberá tenerse en cuenta que tampoco son en sí mismos evidencia de una buena o mala gestión financiera. Si bien en principio, la generación de efectivo procedente de las actividades de inversión que no provenga del cobro de dividendos, podría ser considerada como desfavorable, no lo sería si los fondos obtenidos proceden de la venta de activos improductivos y aplicados posteriormente a la adquisición de activos fijos o de tecnología avanzada.

Estos ejemplos, nos llevan a afirmar que para poder arribar a conclusiones sustentables mediante 
el análisis del estado de flujo de efectivo y de los ratios que se obtengan a partir de la información contenida en él y en el estado de resultados, debemos necesariamente enmarcarlo en el contexto económico y comercial de la empresa, en el ciclo de vida que está atravesando y relacionarlo con la información contenida en otros estados contables. Y a su vez, llevar a cabo un estudio exhaustivo de la vinculación de los flujos de efectivo generados por las distintas actividades entre sí.

Efectuadas estas consideraciones y señaladas las limitaciones, nos parece útil calcular determinados ratios propuestos en diversos trabajos, en el marco de un caso que planteamos a continuación.

\section{Planteo y análisis de un caso}

A continuación, desarrollamos un caso mediante el cual:

- efectuamos presentación del estado de flujo de efectivo mediante método directo, explicitando los cálculos de ingresos y salidas de efectivo en el cuerpo del estado;

- exponemos el estado por método directo, detallando los cálculos de ingresos y salidas de efectivo mediante la presentación de notas complementarias;
- efectuamos un análisis vertical de las cifras del estado;

- realizamos un estudio horizontal, considerando las variaciones de los dos últimos períodos;

- calculamos ratios relevantes de las cifras del estado y de éstas en relación con las del estado de resultados;

- exponemos las conclusiones que surgen de un análisis exhaustivo de las cifras del estado y de su vinculación con el resto de la información disponible, poniendo de manifiesto las razones por las cuales una situación que en un análisis preliminar podría definirse como favorable financieramente, resulta poner en evidencia una situación financiera de gravedad, atento a las causas generadoras de efectivo y a las proyecciones y compromisos de fondos en relación con los periodos futuros.

Se presentan a continuación los estados contables de un ente que desarrolla una actividad comercial, con información comparativa correspondiente al ejercicio inmediato anterior.

El estado de flujo de efectivo ha sido confeccionado por los métodos directo e indirecto previstos en las normas contables locales y luego desarrollaremos dos propuestas alternativas para la exposición de este estado contable. 


\section{Estado de Situación Patrimonial}

\begin{tabular}{|c|c|c|c|c|c|}
\hline \multirow[t]{2}{*}{ ACTIV0 } & Ejercicio & Ejercicio & PASIV0 & Ejercicio & Ejercicio \\
\hline & Corriente & Anterior & & Corriente & Anterior \\
\hline \multicolumn{3}{|l|}{ Activo corriente } & \multicolumn{3}{|l|}{ Pasivo corriente } \\
\hline Caja y Bancos & 15.680 & 10.000 & Deudas comerciales & 45.980 & 20.000 \\
\hline Créditos por Vtas & 30.000 & 26.000 & Préstamos & 17.000 & 31.000 \\
\hline Bienes de cambio & 58.000 & 50.000 & Rem. y cargas sociales & 50.000 & 38.000 \\
\hline \multirow[t]{3}{*}{ Total Activo corriente } & 103.680 & 86.000 & Cargas fiscales IIGG & & 1.680 \\
\hline & & & Cargas fiscales otras & 15.520 & 12.320 \\
\hline & & & Dividendos a pagar & 3.120 & 0 \\
\hline Activo no corriente & 73.200 & 80.000 & Total Pasivo corriente & 131.620 & 103.000 \\
\hline Bienes de uso & 73.200 & 80.000 & & & \\
\hline \multirow[t]{5}{*}{ Total Activo no cte } & & & \multicolumn{3}{|l|}{ Pasivo no corriente } \\
\hline & & & Deudas financieras & 0 & 17.000 \\
\hline & & & Total Pasivo no corriente & 0 & 17.000 \\
\hline & & & Total Pasivo & 131.620 & 120.000 \\
\hline & & & Total PN (s/estado respectivo) & 45.260 & 46.000 \\
\hline \multirow[t]{17}{*}{ Total Activo } & 176.880 & 166.000 & Total Pasivo + PN & 176.880 & 166.000 \\
\hline & Estado de & & & $\overline{\text { CORRIENTE }}$ & $\overline{\text { ANTERIOR }}$ \\
\hline & \multicolumn{3}{|c|}{ Ventas netas } & 69.000 & 63.500 \\
\hline & \multicolumn{3}{|c|}{ CMV } & -40.000 & -30.000 \\
\hline & \multicolumn{3}{|c|}{ Resultado bruto } & 29.000 & 33.500 \\
\hline & \multicolumn{3}{|c|}{ Sueldos y cargas sociales } & -18.000 & -15.714 \\
\hline & \multicolumn{3}{|c|}{ Amortizaciones } & -1.800 & -1.800 \\
\hline & \multicolumn{3}{|c|}{ Impuestos y tasas } & -3.200 & -2.500 \\
\hline & \multicolumn{3}{|c|}{ Otros gastos operativos } & -8.520 & -4.686 \\
\hline & \multicolumn{3}{|c|}{ Resultado operativo } & -2.520 & 8.800 \\
\hline & \multicolumn{3}{|c|}{ Resultados financieros generados por pasivos } & -3.600 & -3.000 \\
\hline & \multicolumn{3}{|c|}{ Otros ingresos - Rdo vta bienes de uso } & 1.500 & -1.000 \\
\hline & \multicolumn{3}{|c|}{ Resultado ord a/ Impuesto a las Ganancias } & -4.620 & 4.800 \\
\hline & \multicolumn{3}{|c|}{ Resultado extraordinario (siniestro mercaderías) } & -3.000 & \\
\hline & \multicolumn{3}{|c|}{ Resultado a/ Impuesto a las Ganancias } & -7.620 & 4.800 \\
\hline & \multicolumn{3}{|c|}{ Impuesto a las Ganancias } & 0 & -1.680 \\
\hline & \multicolumn{3}{|c|}{ PÉRDIDA (GANANCIA) } & -7.620 & 3.120 \\
\hline
\end{tabular}


El impuesto a las ganancias fue reconocido por el método del impuesto a pagar por el ejercicio.

Estado de Evolución del Patrimonio Neto

\begin{tabular}{|c|c|c|c|c|c|c|}
\hline \multirow[t]{3}{*}{$\begin{array}{l}\text { DETALLE } \\
\end{array}$} & \multicolumn{2}{|c|}{ APORTE DE LOS PROPIETARIOS } & \multicolumn{2}{|c|}{ RESULTADOS ACUMULADOS } & \multirow{3}{*}{$\begin{array}{l}\text { TOTAL PN } \\
\text { EJERCIIIO } \\
\text { CORRIENTE }\end{array}$} & \multirow{3}{*}{$\begin{array}{l}\text { TOTAL PN } \\
\text { EJERCICIO } \\
\text { ANTERIOR }\end{array}$} \\
\hline & CAPITAL SOCIAL & APORTES & RESERVA & RESULTADOS & & \\
\hline & & IRREVOCABLES & LEGAL & NO ASIGNADOS & & \\
\hline Saldos iniciales & 12.000 & 0 & 2.400 & 31.600 & 46.000 & 42.880 \\
\hline Aporte socios & & 10.000 & & & 10.000 & \\
\hline Asamblea dist. Rdos & & & & -3.120 & -3.120 & \\
\hline Resultado del ejercicio & & & & -7.620 & -7.620 & 3.120 \\
\hline Totales & 12.000 & 10.000 & 2.400 & 20.860 & 45.260 & 46.000 \\
\hline
\end{tabular}

Estado de flujo de efectivo - método indirecto

\begin{tabular}{lrrr}
\hline VARIACIÓN DEL EFECTIVO Y EQUIVALENTES (NOTA 4) & CORRIENTE & ANTERIOR \\
\hline Efectivo al inicio & 10.000 & 13.500 \\
Efectivo al cierre & 15.680 & 10.000 \\
\hline Aumento (disminución) neto del efectivo & & -3.680 \\
\hline Causas de las variaciones & & \\
Actividades operativas & -4.620 & 4.800 \\
Resultado ordinario a/impuesto a las ganancias & 3.600 & 3.000 \\
Intereses soportados & & \\
Ajustes para arribar al flujo de efectivo por act op ord & 1.800 & 1.800 \\
Amortizaciones bienes de uso & -1.500 & 1.000 \\
Resultado por venta bienes de uso & & \\
Variaciones de activos y pasivos operativos: & -4.000 & -3.500 \\
Aumento créditos por ventas & -18.000 & -25000 \\
Aumento bienes de cambio & 25.980 & 1.686 \\
Aumento deudas comerciales & 12.000 & -14.286 \\
Aumento (disminución) rem y cs sociales a pagar & 3.200 & -20.000 \\
Aumento (disminución) cargas fiscales & & \\
Pago de intereses & -3.600 & -3.000 \\
Pago de impuesto a las ganancias & -1.680 & 0 \\
Flujo de efectivo originado por actividades operativas ordinarias & 13.180 & -53.500 \\
\hline [cont. pág. sgte.] & &
\end{tabular}


[continuación]

Resultado extraordinario a/impuesto a las ganancias

$-3.000$

Variaciones de activos y pasivos operativos

Disminución bienes de cambio

10.000

0

Flujo de efectivo originado por actividades operativas extraordinarias

7.000

0

Flujo de efectivo originado por actividades operativas

$20.180 \quad-53.500$

Actividades de inversión

Cobro por venta de bienes de uso

$6.500-2.000$

Flujo de efectivo generado por actividades de inversión

$6.500 \quad 2.000$

Actividades de financiación

Aportes de los propietarios

$10.000 \quad 0$

Nuevos préstamos

$0 \quad 48.000$

Pago de préstamos

Flujo de efectivo originado por actividades de financiación

$-31.000$

0

Aumento neto del efectivo

$5.680-3.500$

Estado de flujo de efectivo - método directo

\begin{tabular}{|c|c|c|}
\hline VARIACIÓN DEL EFECTIVO Y EQUIVALENTES (NOTA 4) & CORRIENTE & ANTERIOR \\
\hline Efectivo al inicio & 10.000 & 13.500 \\
\hline Efectivo al cierre & 15.680 & 10.000 \\
\hline Aumento (disminución) neto del efectivo & 5.680 & -3.500 \\
\hline \multicolumn{3}{|l|}{ Causas de las variaciones } \\
\hline \multicolumn{3}{|l|}{ Actividades operativas (*) } \\
\hline Cobro por venta de bienes (Nota 5.1) & 65.000 & 60.000 \\
\hline Pago a proveedores de bienes y servicios (Nota 5.2) & -40.540 & -58.000 \\
\hline Pagos al personal (Nota 5.3) & -6.000 & -30.000 \\
\hline Pago de intereses (Nota 5.4) & -3.600 & -3.000 \\
\hline Pagos de impuesto a las ganancias (Nota 5.5) & -1.680 & 0 \\
\hline Pagos de otros impuestos (Nota 5.6) & 0 & -22.500 \\
\hline Flujo de efectivo generado por actividades operativas ordinarias & 13.180 & -53.500 \\
\hline Cobro seguro por siniestro & 7.000 & 0 \\
\hline Flujo de efectivo generado por actividades operativas extraord & 7.000 & \\
\hline Flujo de efectivo originado por actividades operativas & 20.180 & -53.500 \\
\hline \multicolumn{3}{|l|}{ Actividades de inversión } \\
\hline Cobro por venta de bienes de uso & 6.500 & 2.000 \\
\hline Flujo de efectivo generado por actividades de inversión & 6.500 & 2.000 \\
\hline
\end{tabular}




\begin{tabular}{l} 
[continuación] \\
\hline Actividades de financiación \\
Aportes de los propietarios \\
Nuevos préstamos \\
Pago de préstamos \\
\hline Flujo de efectivo aplicado (originado por) actividades de financiación
\end{tabular}

Algunas conclusiones que surgen de la lectura de los estados presentados son:

1. En el ejercicio corriente pese a haberse registrado una pérdida operativa, el flujo de efectivo de las actividades operativas muestra una generación de fondos, que permitió afrontar en una parte importante el flujo de efectivo aplicado a las actividades de financiación. En el ejercicio anterior la situación había sido la inversa, mostrando una ganancia operativa y un flujo de efectivo aplicado en las actividades operativas.

2. Las razones que explican las situaciones descriptas en el punto 1 no pueden ser brindadas observando únicamente el estado de flujo de efectivo por el método directo, mientras que sí las podemos encontrar en el estado preparado bajo el método indirecto, donde se muestran todas las partidas que se suman 0 deducen al resultado ordinario (en su caso también desde el extraordinario) para arribar al flujo de efectivo de las actividades operativas. En el ejercicio corriente puede notarse una disminución en el capital operativo (activos operativos menos pasivos operativos) como principal causa, conjuntamente con los fondos provenientes de operaciones extraordinarias, lo que explica por qué fue posible la generación de fondos por las actividades operativas a pesar de que el resultado operativo había sido una pérdida.

A continuación, compartimos dos alternativas de presentación del estado de flujo de efectivo bajo el método directo, que tienen como objetivo combinar sus ventajas con las provenientes del método indirecto:

\section{Alternativa 1. Estado de flujo de Efectivo por método directo con notas aclaratorias que muestran cómo se determinan las principales clases de entradas y salidas brutas de efectivo} Consiste en la presentación del estado de flujo de efectivo confeccionado por el método directo, pero con el agregado de la referencia a notas incluidas en la información complementaria, que detallan los cálculos confeccionados para determinar los ingresos y salidas de fondos reflejados en el cuerpo del estado.

Las notas en el caso planteado podrían redactarse del siguiente modo: 


\begin{tabular}{|c|c|c|}
\hline & CORRIENTE & ANTERIOR \\
\hline \multicolumn{3}{|l|}{ Nota 5.1: Cobro por ventas } \\
\hline Ventas pendientes de cobro al inicio del ejercicio & 26.000 & 22.500 \\
\hline Ventas devengadas en el periodo & 69.000 & 63.500 \\
\hline \multirow[t]{2}{*}{ Ventas pendientes de cobro al cierre del ejercicio } & -30.000 & -26.000 \\
\hline & 65.000 & 60.000 \\
\hline \multicolumn{3}{|l|}{ Nota 5.2: Pagos a proveedores de bienes y servicios } \\
\hline Deudas comerciales al inicio del periodo & 20.000 & 18.314 \\
\hline Compras de bienes de cambio del ejercicio & 58.000 & 55.000 \\
\hline Otros gastos operativos del ejercicio & 8.520 & 4.686 \\
\hline \multirow[t]{2}{*}{ Deudas comerciales al cierre del periodo } & -45.980 & -20.000 \\
\hline & 40.540 & 58.000 \\
\hline \multicolumn{3}{|l|}{ Nota 5.3: Pagos al personal } \\
\hline Sueldos y cargas sociales adeudadas al inicio del periodo & 38.000 & 52.286 \\
\hline Sueldos y cargas sociales devengadas durante el ejercicio & 18.000 & 15.714 \\
\hline \multirow[t]{2}{*}{ Sueldos y cargas sociales adeudadas al cierre del periodo } & -50.000 & -38.000 \\
\hline & 6.000 & 30.000 \\
\hline \multicolumn{3}{|l|}{ Nota 5.4: Pago de intereses } \\
\hline Intereses a pagar al inicio del ejercicio & 0 & 0 \\
\hline Intereses devengados en el ejercicio & 3.600 & 3.000 \\
\hline \multirow[t]{2}{*}{ Intereses a pagar al c ierre del ejercicio } & 0 & 0 \\
\hline & 3.600 & 3.000 \\
\hline \multicolumn{3}{|l|}{ Nota 5.5: Pago impuesto a las ganancias } \\
\hline Deudas imp gcias al inicio del periodo & 1.680 & 0 \\
\hline Impuesto devengado en el ejercicio & 0 & 1.680 \\
\hline \multirow[t]{2}{*}{ Deudas imp gcias al cierre del periodo } & 0 & -1.680 \\
\hline & 1.680 & 0 \\
\hline \multicolumn{3}{|l|}{ Nota 5.6: Pagos de otros impuestos } \\
\hline Deudas impositivas al inicio del periodo & 12.320 & 32.320 \\
\hline Impuestos devengados en el ejercicio & 3.200 & 2.500 \\
\hline \multirow[t]{2}{*}{ Deudas impositivas al cierre del periodo } & -15.520 & -12.320 \\
\hline & 0 & 22.500 \\
\hline
\end{tabular}

\section{Alternativa 2. Estado de flujo de Efectivo por método directo con cálculos sobre determinación de entradas y salidas de efectivo en cuerpo del estado}

Consiste en la presentación del estado de flujo de efectivo reflejando en el cuerpo del mismo los resultados y variaciones de activos y pasivos que determinan las entradas y salidas de fondos ocurridas durante el periodo. 


\begin{tabular}{|c|c|c|c|}
\hline \multirow{2}{*}{ Variación del efectivo y equivalentes } & \multicolumn{2}{|c|}{ CORRIENTE } & \multirow[t]{2}{*}{ ANTERIOR } \\
\hline & & & \\
\hline Efectivo al inicio & & 10.000 & 13.500 \\
\hline Efectivo al cierre & & 15.680 & 10.000 \\
\hline Aumento (disminución) neto del efectivo & & 5.680 & -3.500 \\
\hline \multicolumn{4}{|l|}{ Causas de las variaciones } \\
\hline \multicolumn{4}{|l|}{ Actividades operativas } \\
\hline Ventas del periodo & 69.000 & & 63.500 \\
\hline Aumento créditos por ventas & -4.000 & & -3.500 \\
\hline Cobros por ventas & & 65.000 & 60.000 \\
\hline Compras de bienes de cambio y gastos operativos & -66.520 & & -59.686 \\
\hline Aumento de deudas comerciales & 25.980 & & 1.686 \\
\hline Pago a proveedores de bienes y servicios & & -40.540 & -58.000 \\
\hline Sueldos y cargas sociales del ejercicio & -18.000 & & -15.714 \\
\hline Aumento (disminución) rem y cs sociales a pagar & 12.000 & & -14.286 \\
\hline Pagos al personal & & -6.000 & -30.000 \\
\hline Intereses devengados en el ejercicio & -3.600 & & -3.000 \\
\hline Variación deudas por intereses & 0 & & 0 \\
\hline Pago de intereses & & -3.600 & -3.000 \\
\hline Impuesto a las Ganancias del periodo & 0 & & -1.680 \\
\hline Disminución (aumento) deuda imp gcias & -1.680 & & 1.680 \\
\hline Pago imp gcias & & -1.680 & 0 \\
\hline Otros impuestos devengados en el periodo & -3.200 & & -2.500 \\
\hline Aumento otras de deudas impositivas & 3.200 & & -20.000 \\
\hline Pago de otros impuestos & & 0 & -22.500 \\
\hline Flujo de efectivo originado por act. operativas ord. & & 13.180 & -53.500 \\
\hline Cobro seguro por siniestro & & 7.000 & 0 \\
\hline Flujo de efectivo originado por act. operativas extraord. & & 7.000 & \\
\hline Flujo de efectivo originado por actividades operativas & & 20.180 & -53.500 \\
\hline Actividades de inversión & & & \\
\hline Cobro por venta de bienes de uso & & 6500 & 2000 \\
\hline Flujo de efectivo generado por actividades de inversión & & 6500 & 2000 \\
\hline
\end{tabular}




\section{[continuación]}

Actividades de financiación

Aportes de los propietarios

Nuevos préstamos

Pago de préstamos

Flujo de efectivo originado por act. de financiación

Aumento (disminución) neto del efectivo

\begin{tabular}{rrr}
\hline CORRIENTE & & ANTERIOR \\
& 10.000 & 0 \\
0 & 48.000 \\
\hline-31.000 & & 0 \\
\hline-21.000 & & 48.000 \\
\hline & & \\
\hline
\end{tabular}

\section{Análisis del estado de flujo de efectivo}

Si bien el método directo no es suficiente para proporciona información más clara que el método evaluar por sí solo la situación financiera del ente y la vinculación con sus causales económicas, ejemplos.

\begin{tabular}{|c|c|c|}
\hline ORÍGENES & & \\
\hline Cobro por venta de bienes & 65.000 & $73,45 \%$ \\
\hline Cobro seguro por siniestro & 7.000 & $7,91 \%$ \\
\hline Cobro por venta de bienes de uso & 6.500 & $7,34 \%$ \\
\hline Aportes de los propietarios & 10.000 & $11,30 \%$ \\
\hline Total orígenes & 88.500 & \\
\hline \multicolumn{3}{|l|}{ APLICACIONES } \\
\hline Pago a proveedores de bienes y servicios & -40.540 & $48,95 \%$ \\
\hline Pagos al personal & -6.000 & $7,24 \%$ \\
\hline Pago de intereses & -3.600 & $4,35 \%$ \\
\hline Pagos de impuesto a las ganancias & -1.680 & $2,03 \%$ \\
\hline Pago de préstamos & -31.000 & $37,43 \%$ \\
\hline Total aplicaciones & -82.820 & \\
\hline Aumento del efectivo & 5.680 & \\
\hline
\end{tabular}

Al realizar un análisis vertical agrupando en forma separada las entradas de las salidas de fondos, es posible determinar el grado de importancia que cada una de ellas representa en relación con el total de fondos originados y el total aplicado respectivamente.

En este caso se observa que la principal fuente de ingresos es el cobro por ventas. También se generaron fondos por aportes de los propietarios, por actividades extraordinarias que no se espera se repetirán y mediante la venta de bienes de uso.

En relación con los pagos, el análisis evidencia una fuerte preponderancia de los egresos dirigidos a proveedores de bienes y servicios y le siguen en importancia la salida de fondos destinadas a la devolución de préstamos y en una medida mucho menor el pago sueldos y cargas, de intereses y de impuesto a las ganancias. 
Puede resultar interesante para el análisis de la evolución financiera del ente, la comparación con los porcentuales obtenidos en el periodo anterior.

\section{Ejercicio anterior}

\begin{tabular}{|c|c|c|}
\hline ORÍGENES & & \\
\hline Cobro por venta de bienes & 60.000 & $54,55 \%$ \\
\hline Cobro por venta de bienes de uso & 2.000 & $1,82 \%$ \\
\hline \multirow[t]{2}{*}{ Nuevos préstamos } & 48.000 & $43,64 \%$ \\
\hline & 110.000 & \\
\hline \multicolumn{3}{|l|}{ APLICACIONES } \\
\hline Pago a proveedores de bienes y servicios & -58.000 & $51,10 \%$ \\
\hline Pagos al personal & -30.000 & $26,43 \%$ \\
\hline Pago de intereses & -3.000 & $2,64 \%$ \\
\hline \multirow[t]{2}{*}{ Pagos de otros impuestos } & -22.500 & $19,82 \%$ \\
\hline & -113.500 & \\
\hline Disminución del efectivo & -3.500 & \\
\hline
\end{tabular}

Como puede notarse, en el ejercicio anterior el ente recurrió al financiamiento de terceros, lo que generó fondos en una proporción muy cercana a los obtenidos por cobros por ventas. Por otra parte, las dificultades financieras han llevado a la necesidad de enajenar bienes de uso. Ha de observarse que estos fondos fueron empleados para el sostenimiento de egresos operativos (pago a proveedores, al personal y de impuestos) y no para la incorporación de inversiones o nuevos bienes de uso. Esto refleja que las dificultades financieras del ente se sostienen desde el periodo anterior.

A los fines de evaluar la evolución financiera resulta también interesante efectuar un análisis horizontal:

\section{Análisis horizontal}

\begin{tabular}{|c|c|c|c|}
\hline & CORRIENTE & ANTERIOR & VARIACIÓN \\
\hline Cobro por venta de bienes & 65.000 & 60.000 & $8,33 \%$ \\
\hline Pago a proveedores de bienes y servicios & -40.540 & -58.000 & $-30,10 \%$ \\
\hline Pagos al personal & -6.000 & -30.000 & $-80,00 \%$ \\
\hline Pago de intereses & -3.600 & -3.000 & $20,00 \%$ \\
\hline Pagos de impuesto a las ganancias & -1.680 & 0 & $\mathrm{E}$ \\
\hline Pagos de otros impuestos & 0 & -22.500 & $-100,00 \%$ \\
\hline Cobro por venta de bienes de uso & 6.500 & 2.000 & $225,00 \%$ \\
\hline Aportes de los propietarios & 10.000 & 0 & $\underline{E}$ \\
\hline Nuevos préstamos & 0 & 48.000 & $-100,00 \%$ \\
\hline Pago de préstamos & -31.000 & 0 & $\mathrm{E}$ \\
\hline
\end{tabular}


Al analizar la variación de los movimientos de fondos entre ambos periodos, puede observarse que existió un aumento del pago de intereses y se incorporó el pago del impuesto a las ganancias y de préstamos.

Estas disminuciones de fondos fueron financiadas mediante un leve aumento del cobro por ventas, la reducción de pagos a proveedores, al personal y de otros impuestos y mediante el aporte de los propietarios y el incremento de cobros por nuevas ventas de bienes de uso.

Por otra parte, existe una gran diversidad de ratios que es posible calcular con la información que surge del estado de flujo de efectivo, a los fines de realizar un análisis exhaustivo de la situación financiera del ente y efectuar proyecciones. No obstante, en cualquier caso, los resultados de cada uno de estos ratios proporcionan información parcial que necesariamente debe ser considerada teniendo en cuenta el contexto comercial y económico en el que se desenvuelve el ente y deben evaluarse en forma conjunta con otros indicadores y datos obtenidos de otros estados.

La doctrina propone una variedad de ratios vinculados al estado de flujo de efectivo. A continuación, aplicamos algunos de ellos al caso planteado y las conclusiones que pueden extraerse.

En primer lugar, resulta importante destacar que en el caso desarrollado se observa que parte de los flujos operativos tienen el carácter de extraordinarios, es decir que son de suceso infrecuente. En consecuencia, sugerimos que al momento de estimar estos indicadores dichos flujos sean segregados, de modo tal que los resultados que se obtengan sean comparables con los observados en periodos anteriores y de este modo puedan realizarse proyecciones razonables.

Ratio indicador de la importancia de los flujos operativos en la generación del flujo de efectivo total:

\begin{tabular}{ll}
\hline RATIO & CÁLCUL0 \\
\hline Flujo de efect generado por actividades operativas ordinarias & $\frac{13.180}{\text { RESULTAD0 }}$ \\
\hline Flujo de efectivo total & $\frac{2,32}{5.680}$ \\
\hline
\end{tabular}

Este ratio indica que las actividades operativas ordinarias contribuyeron de forma significativa en la obtención del flujo de efectivo. Sin embargo, la información que brinda por sí solo resulta escasa y no es indicativa de la situación financiera del ente, por lo que debe complementarse con el análisis de otras cuestiones.

Ratio indicador de la medida en que los flujos operativos contribuyeron para afrontar los flujos por actividades de financiación: \begin{tabular}{l} 
RATIO \\
Flujo de efectivo aplicado a actividades de financiación \\
\hline Flujo generado por actividades operativas ordinarias \\
\hline
\end{tabular}

En este caso se observa que el flujo de efectivo generado por las actividades operativas ordinarias del ente no fue suficiente para cubrir las salidas de fondos vinculadas a actividades de financiación, por lo que fue necesario recurrir a la venta de bienes de uso (fondos originados por actividades de inver- sión) y otra parte fue financiada mediante cobros operativos extraordinarios.

Ratio indicador de la medida en que los flujos de actividades de inversión contribuyeron para afrontar los flujos por actividades de financiación: 


\begin{tabular}{l}
\hline RATIO \\
Flujo de efectivo aplicado a actividades de financiación \\
\hline Flujo generado por actividades de inversión \\
Este ratio arroja un resultado del que deriva una \\
conclusión similar a la planteada para el cálculo \\
anterior, es decir, que las aplicaciones financieras \\
fueron cubiertas con orígenes de actividades opera- \\
tivas y de inversión.
\end{tabular}

RATIO

Intereses pagados

Flujo de efect act operativas ordinarias

En el caso planteado los intereses pagados fueron expuestos dentro de las actividades operativas, por

$\begin{array}{r}\hline \text { CÁLCUL0 } \\ \frac{-21.000}{6500} \\ \hline\end{array}$

Ratio indicador de la importancia de los pagos por costos financieros respecto del origen de fondos operativos ordinarios:

\begin{tabular}{|c|c|}
\hline CÁLCULO & RESULTADO \\
\hline-3.600 & $-0,21$ \\
\hline 16.780 & \\
\hline
\end{tabular}

lo cual deben adicionarse al flujo operativo para calcular este ratio.

\section{Razón de cobertura}

\begin{tabular}{llc}
\hline RATI0 & CÁLCUL0 & $\frac{\text { RESULTAD0 }}{13.180}$ \\
\hline Efec operaciones ordinarias & $-0,43$ \\
\hline Efect aplicado a pago deudas financieras & -31.000 & \\
\hline
\end{tabular}

Del cálculo presentado puede observarse que los flujos operativos ordinarios no fueron suficientes para cubrir el pago de las deudas financieras, por lo que fue necesario recurrir a otros recursos.

Indicador de la medida en que el flujo operativo es suficiente para cubrir las deudas del ente

\begin{tabular}{llr}
\hline RATI0 & CÁLCUL0 & $\frac{\text { RESULTADO }}{13.180}$ \\
\hline Efec operaciones ordinarias & 0,10 \\
\hline Pasivo corriente & $\frac{131.620}{-}$ \\
\hline
\end{tabular}

Como puede notarse, el resultado de este ratio es muy bajo. De esto se infiere que el ente no estará en condiciones de afrontar con sus actividades operativas sus pasivos corrientes (exigibles al cierre 0 que se convertirán en exigibles en los doce meses posteriores al cierre), si mantiene un nivel de gene- ración de flujos operativos similar al del corriente ejercicio.

La situación financiera del ente que a simple vista podía parecer favorable debido a la presencia de flujos operativos positivos en el estado de flujo de efectivo, luego de este análisis evidencia un 
panorama de serias dificultades financieras. Esta situación nos obliga a estudiar las causas que la originan, para lo cual podemos recurrir al análisis conjunto de estas cuestiones con los aspectos económicos de la actividad del ente reflejados en su estado de resultados. A continuación, se exponen algunos ratios que consideran para su cálculo movimientos financieros y resultados del periodo:

Relación entre flujos de fondos operativos y el resultado de la actividad principal antes de amortizaciones:

\begin{tabular}{llr}
\hline RATI0 & CÁLCUL0 & $\frac{\text { RESULTAD0 }}{13.180}$ \\
\hline Efec operaciones ordinarias & $-18,31$ \\
\hline Resultado operativ0 + amortizaciones & -720 & \\
\hline
\end{tabular}

El resultado de este ratio nos indica que no existe correlación entre el resultado operativo susceptible de generar fondos (porque se adicionaron las amortizaciones) y los flujos de fondos por actividades operativas ordinarias. Esto guarda relación con lo que comentábamos anteriormente acerca de la incidencia que los incrementos de los pasivos operativos tuvieron en la generación de fondos durante el ejercicio.

Cuanto más cercano es el resultado a 1 , más fuerte es la correlación entre el resultado operativo y sus efectos financieros.

Relación entre cobranzas y ventas:

\begin{tabular}{ll}
\hline RATI0 & CÁLCUL0 \\
\hline Cobranzas & $\frac{\text { RESULTAD0 }}{-000}$ \\
\hline Ventas devengadas & 09.94 \\
\hline
\end{tabular}

En este caso se observa un resultado inferior a 1 y casi idéntico al resultado que arroja el ratio (60 000/63 500) para el periodo anterior. Esto nos indica que el plazo de cobranzas se ha mantenido estable.

Al determinar este indicador resulta importante considerar adecuadamente el IVA, es decir, que si las cobranzas en el numerador incluyen este impuesto, en el denominador deben tomarse en cuenta las ventas con IVA incluido 0 el IVA debe excluirse del numerador y denominador. En nuestra opinión los cobros y pagos deben exponerse sin incluir el IVA, exponiendo por separado el efecto en el flujo de efectivo que dicho tributo haya generado. En igual sentido se ha expresado la FACPCE (2009:19, 55-59). Idénticas consideraciones sobre el tratamiento del IVA resultan aplicables al ratio que se indica a continuación.

Relación entre pagos a proveedores y compras:

\begin{tabular}{|c|c|c|}
\hline RATIO & $\begin{array}{r}\text { CÁLCULO } \\
\text { EJERCICIO } \\
\text { CORRIENTE }\end{array}$ & $\begin{array}{r}\text { RESULTADO } \\
\text { EJERCICIO } \\
\text { CORRIENTE }\end{array}$ \\
\hline Pagos a prov. bs y scios. & -40.540 & $-0,61$ \\
\hline Compras + gastos operativos & 66.520 & \\
\hline RATIO & $\begin{array}{r}\text { CÁLCULO } \\
\text { EJERCICIO } \\
\text { ANTERIOR }\end{array}$ & $\begin{array}{r}\text { RESULTADO } \\
\text { EJERCICIO } \\
\text { ANTERIOR }\end{array}$ \\
\hline Pagos a prov. bs y scios. & -58.000 & $-0,97$ \\
\hline Compras + gastos operativos & 59.686 & \\
\hline
\end{tabular}

En ambos ejercicios se observa que la relación arroja un resultado inferior a la unidad. Sin embargo, en el ejercicio corriente el valor es notablemente menor a 1. Este comportamiento se debe a que aumen- 
taron las compras durante el ejercicio corriente y a una reducción de los pagos respecto del periodo anterior, lo que evidencia un prolongamiento en los plazos de financiación.

Relación entre pagos de sueldos y cargas sociales y estos gastos devengados

\begin{tabular}{|c|c|c|}
\hline RATIO & $\begin{array}{r}\text { CÁLCULO } \\
\text { EJERCICIO } \\
\text { CORRIENTE }\end{array}$ & $\begin{array}{r}\text { RESULTADO } \\
\text { EJERCICIO } \\
\text { CORRIENTE }\end{array}$ \\
\hline Pago de sueldos y cs. sociales & -6.000 & 0,33 \\
\hline Sueldos y cargas devengados & -18.000 & \\
\hline RATIO & $\begin{array}{r}\text { CÁLCULO } \\
\text { EJERCICIO } \\
\text { ANTERIOR }\end{array}$ & $\begin{array}{r}\text { RESULTADO } \\
\text { EJERCICIO } \\
\text { ANTERIOR }\end{array}$ \\
\hline Pago de sueldos y cs. sociales & -30.000 & 1,91 \\
\hline Sueldos y cargas devengados & -15.714 & \\
\hline
\end{tabular}

En relación con estos gastos se observa que en el ejercicio anterior el ratio arrojó un valor superior a la unidad, lo que indica que durante dicho periodo se abonaron deudas con el personal provenientes de ejercicios anteriores.

En el ejercicio corriente, en cambio, el resultado es menor que 1 , lo cual responde a parcialmente a un incremento de los sueldos devengados y principalmente a una reducción de las erogaciones realizadas por estos conceptos. Esto indica que el ente ha alargado los plazos de pago de estas obligaciones, lo que ha contribuido a la generación de un flujo operativo positivo, a pesar de la presencia de un resultado operativo negativo.

\section{Conclusiones}

A lo largo del presente trabajo, hemos puesto de manifiesto que, a los fines de efectuar proyecciones y análisis financieros, la información contenida en el estado de flujo de efectivo es de fundamental importancia, pero no es suficiente, aun mediante el análisis de sus causas, y debe necesariamente, complementarse con la consideración de los saldos de los activos y pasivos operativos vinculados. Es en este punto que cobra relevancia el método seleccionado para brindar información en relación con los flujos de efectivo y equivalentes de efectivo generados por las actividades operativas.

Si bien el método directo es el que permite una lectura y comprensión sencilla por parte del usuario, en particular a los fines de realizar proyecciones, no resulta ser suficiente para el análisis de la vinculación entre el resultado obtenido por el ente y la generación de fondos del periodo. Por ello, resulta necesario recurrir a su estudio conjunto con los saldos patrimoniales relacionados. A los fines de que la información presentada permita esta vinculación, proponemos incluir información en el cuerpo del estado de flujo de efectivo 0 en una nota complementaria, sobre cómo se determinan las distintas clases de entradas y salidas brutas de efectivo que se exponen por el método directo, partiendo de los resultados devengados y considerando los aumentos o disminuciones de los activos y pasivos operativos vinculados con los mismos. De esta manera, se pone de manifiesto la relación de los estados de resultados y de flujo de efectivo, considerando que la apertura de la información propuesta es el puente que los vincula, quizás con mayor claridad que la requerida por el método indirecto. Asimismo, permite mejorar sustancialmente el análisis y la proyección a efectuar, teniendo en cuenta la incidencia de los saldos de los rubros patrimoniales vinculados. En definitiva, permite poner de manifiesto las ventajas de ambos métodos.

En la medida en que la información se presente mediante el método directo, además, es posible efectuar un análisis vertical del estado de flujo de efectivo determinando la incidencia o participación de las distintas partidas del estado en relación con los subtotales o totales del mismo. De la misma manera, podríamos realizar un estudio horizontal 0 de evolución de las partidas a lo largo de dos 0 más periodos. Algunos autores proponen también el cálculo de ratios vinculando entre sí los distintos 
subtotales de este estado y con algunos del estado de resultados. En el ejemplo desarrollado, efectuamos el cálculo de los ratios más relevantes y procedemos a su análisis en el contexto del resto de la información de la que se dispone.

Para poder arribar a conclusiones razonables mediante el análisis del estado de flujo de efectivo y de los ratios que se obtengan a partir de la información contenida en él y en el estado de resultados, debemos necesariamente dar consideración al contexto económico y comercial de la empresa, al momento en el ciclo de su vida que está atravesando y a la información contenida en otros estados contables.

\section{Referencias bibliográficas}

- Consejo Emisor de Normas Internacionales de Contabilidad (IASB). (2015). Norma Internacional de Información Financiera para PYMES. Recuperado en Julio 2017 de https://www.facpce.org.ar/pdf/niifpymes2015.pdf

- _ (2016). Norma Internacional de Contabilidad (NIC) No 1 , Presentación de Estados Financieros. Recuperado en Julio 2017 de http://www.normasinternacionalesdecontabilidad.es/nic/pdf/nic01.pdf

- Federación Argentina de Consejos Profesionales en Ciencias Económicas (FACPCE) (2008). Interpretación $N^{\circ}$ 2: Estado de Flujo de Efectivo y sus equivalentes. Recuperado en Julio 2017 de http://www.facpce. org.ar:8080/infopro/categorias. php?categoria $=2$
- - (2009). Informe № 29: Estado de flujo de efectivo. Recuperado en Julio 2017 de https://www.facpce. org.ar/pdf/cecyt/contabilidad-29.pdf

- Fowler Newton, E. (2001, diciembre). Análisis del estado de flujo de efectivo. Enfoques Contabilidad y Administración, 7-24. Buenos Aires: La Ley.

- (2011). Cuestiones Contables Fundamentales. Buenos Aires: La Ley.

- García Fronti, I. (2003, enero). Estado de flujo de efectivo: su utilidad en la toma de decisiones a partir del ciclo de vida de las empresas. Enfoques Contabilidad y Administración, 1, 46-52. Buenos Aires: La Ley.

- Pérez, J.0. (2008, marzo). El Estado de Flujo de Efectivo y la administración financiera. Enfoques Contabilidad y Administración. Buenos Aires: La Ley.
- Rondi, G.R., Casal, M.C., Galante, M.J. y Gómez, M. (2014). Normas para la presentación de estados contables en Argentina. Trabajo presentado en las XXXV Jornadas Universitarias de Contabilidad. Facultad de Ciencias Económicas y Empresariales, Universidad Católica de Cuyo. 24, 25 y 26 de septiembre de 2014, San Juan, Argentina.

- Rondi, G.R., y Galante, M.J. (2007). La EBITDA como indicador de desempeño. Un análisis crítico. Trabajo presentado en las XXVIII Jornadas Universitarias de Contabilidad. Facultad de Ciencias Económicas, Universidad Nacional de Mar del Plata. 07, 08 y 09 de noviembre 2007, Mar del Plata, Argentina.

\section{Registro bibliográfico}

Rondi, G.R., Casal, M.C., Galante, M.J., y Gómez, M. (2017). Reflexiones sobre el estado de flujo de efectivo e ideas para su presentación por el método directo. Revista Ciencias Económicas, 14(01), 99-121. 